\title{
Quercetin Enhances Cisplatin Sensitivity of Human Osteosarcoma Cells by Modulating microRNA-217-KRAS Axis
}

\author{
Xian Zhang, Qinggong Guo, Jingtao Chen, and Zhaohui Chen*
}

\begin{abstract}
Quercetin can suppress osteosarcoma cell growth and metastasis. However, other effects of quercetin on osteosarcoma remain largely unknown. This research aims to evaluate the effects of quercetin in combination with cisplatin as treatment for osteosarcoma and investigate its regulatory mechanism. Cell viability and apoptosis in 143B cell line were determined after treatment with quercetin and/or cisplatin. RT-PCR and Western blot analysis were performed to determine the RNA or protein expression levels. Moreover, transwell assay was used to evaluate metastasis. Furthermore, rescue experiments were performed to investigate the potential regulatory mechanism of the treatment. Results showed that quercetin with concentration that was equal to or greater than $10 \mu \mathrm{M}$ inhibited 143B proliferation, while $5 \mu \mathrm{M}$ quercetin enhanced the cisplatin sensitivity of $143 \mathrm{~B}$ cells. Expression of miR217 was upregulated after quercetin and/or cisplatin treatment, while its target KRAS was downregulated both at mRNA and protein levels. MiR-217 knockdown led to the loss of enhanced cisplatin sensitivity while miR-217 overexpression showed the opposite effects, indicating that quercetin regulated cisplatin sensitivity by modulating the miR-217-KRAS axis. In conclusion, $5 \mu \mathrm{M}$ quercetin enhanced the cisplatin sensitivity by modulating the miR217-KRAS axis. This finding suggests that quercetin may be administered with cisplatin to improve the treatment for osteosarcoma.
\end{abstract}

\section{INTRODUCTION}

Osteosarcoma is one of the common clinical and multiple primary malignant bone tumor which damages the health of children and young adults. Multi-agent chemotherapy and primary tumor surgical resection are the most common treatments of osteosarcoma (Bielack et al., 2008). However, the common

Department of Orthopaedics, the First Hospital Affiliated of Henan University, Henan, 475000, China

*Correspondence: chenzhaohui1985@126.com

Received 10 February, 2015; revised 14 April, 2015; accepted 23 April 2015; published online 10 June, 2015

Keywords: cisplatin, KRAS, microRNA-217, osteosarcoma, quercetin anti-neoplastic drugs, including cisplatin, doxorubicin, etoposide, ifosfamide, among others cause tolerance to chemotherapeutic agent, as well as acute/long-term toxicity (Bielack et al., 2008).

Quercetin is a flavonoid commonly found in our daily diet. Properties of quercetin, such as its being anti-inflammatory, antioxidant, anti-proliferation, and its promotion of apoptosis on cancer cells were reported (SS, 2012). Thus, owing to its anticancer properties, quercetin might be used as adjunctive therapy with less side effects to enhance cancer chemoprevention (Li et al., 2014; Orsolic and Car, 2014; Wang et al., 2014; Yi et al., 2014). In hepatoma, quercetin enhanced the sensitivity of cancer cells to cisplatin (Kim et al., 2008). However, in ovarian cancer cells, low concentrations of quercetin attenuate the therapeutic effects of cisplatin by reduced production of intracellular reactive oxygen species ( $\mathrm{Li}$ et al., 2014). Studies also showed that quercetin suppresses osteosarcoma cell proliferation (Liang et al., 2011; Suh et al., 2010). However, a few researches on the effects of combined quercetin and chemotherapy on osteosarcoma, especially on their regulatory mechanism were conducted.

In this paper, we aimed to investigate the effects of quercetin combined with cisplatin, a widely used chemotherapeutic agent, as well as to investigate its regulatory mechanism.

\section{MATERIALS AND METHODS}

\section{Cell culture and transfection}

Human osteosarcoma 143B cell line was purchased from the Cell Resource Center of Institute of Basic Medical Sciences, Chinese Academy of Medical Sciences. The 143B cell linewascultured in Dulbecco's modified Eagle medium with $10 \%$ fetal bovine serum (Life Technologies, Germany).

The miR-217 mimic, antagomir, and siRNA of KRAS were purchased from RIBOBIO Co. Ltd (China). miR-217 mimic at $50 \mathrm{nM}$ or $100 \mathrm{nM}$ miR-217 antagomir was used during transfection of $143 \mathrm{~B}$ cells by Lipofectamine 3000 , used according to the manufacturer's instruction (Invitrogen, USA).

Total RNA extraction, single strand cDNA synthesis, and real-time quantitative PCR assays

Trizol method was used for all of the extraction of total RNA in this study (Ambion, USA). For single strand cDNA synthesis, 3 $\mu \mathrm{g}$ RNA was used as template and reverse transcription was performed by M-MLV (Life Science, USA). The use of real-time quantitative PCR (RT-PCR) progress and primer sequences was 
entirely adopted from the previous research (Guo et al., 2014).

Cell proliferation, viability, and apoptosis assay

The CCK-8 kit was used in cell proliferation or viability assay according to the user's instruction (DOJINDO, Japan). Approximately 5000 osteosarcoma cells were incubated at $37^{\circ} \mathrm{C}$ for 2 $\mathrm{h}$ in $10 \%$ CCK-8 reagent, and absorption was measured at 450 $\mathrm{nm}$ using micro-plate reader. For 143B apoptosis assays, apoptosis detection kit was used following the user's instruction (BD Biosciences, USA). Cell apoptosis was detected by $\mathrm{C} 6$ Flow Cytometer (Thermo, USA).

\section{Cell migration and invasion assay}

Transwell assays were performed to evaluate the migration ability of osteosarcoma 143B cells. Approximately $5 \times 10^{4}$ cells suspended in $200 \mu \mathrm{l}$ DMEM medium without FBS was placed on the upper chamber of each transwell insert, while $600 \mu \mathrm{l}$ DMEM with $10 \%$ FBS was placed in the lower chamber (Millipore, USA). After $24 \mathrm{~h}$, migrating cells on the lower surface of the chamber were stained with $10 \%$ crystal violet. For invasion assay, $40 \mu \mathrm{l}$ of $1 \mathrm{mg} / \mathrm{ml}$ matrigel was coated in the bottom of the chamber (Millipore, USA).The remaining processes were similar to that of the other transwell assays.

\section{Quercetin and cisplatin treatment}

Cisplatin was obtained from Sigma (USA). For 143B cell viability assay, 143B cells were treated with0, 2, 4, 6, 8, 10, and 12 $\mu \mathrm{M}$ cisplatin for $24 \mathrm{~h}$. About 5,000 cells were used for cell viability assay. Quercetin was purchased from Sigma (USA), and was dissolved in water with $0.5 \%(\mathrm{v} / \mathrm{v})$ ethanol. Water with $0.5 \%$ ethanol was used for the control. About 20,000 cells treated with $5 \mu \mathrm{M}$ cisplatin and $5 \mu \mathrm{M}$ quercetin were used in the apoptosis assay.

Western blot analysis

Western blot analysis was performed strictly following the method described in our previous research (Guo et al., 2014).

\section{Statistics}

Each of the experiments in this study were performed at least three times. Student's $t$-test (two-tailed) and the $X^{2}$ test were performed, and statistically significant level was set at $\alpha=0.05$ (two-side). Data are presented as mean \pm SD.

\section{RESULTS}

Quercetin equal to or greater than $10 \mu \mathrm{M}$ inhibited viability of 143B osteosarcoma cells

We determined the effect of varying quercetin concentrations on the viability of $143 \mathrm{~B}$ cells. We found that $5 \mu \mathrm{M}$ of quercetin had no significant effect on 143B viability. However, quercetin concentration of $10 \mu \mathrm{M}$ or higher significantly reduced cell viability at $24 \mathrm{~h}$ or $48 \mathrm{~h}$ post-treatment compared with the control group (Fig. 1). This result indicated that high dose of quercetin could inhibit 143B proliferation.

\section{$5 \mu \mathrm{M}$ quercetin enhanced cisplatin sensitivity of 143B}

Cells of $143 \mathrm{~B}$ line were co-treated with $5 \mu \mathrm{M}$ quercetin and cisplatin for $24 \mathrm{~h}$ to determine the effect of quercetin on cisplatin sensitivity. Result revealed that cells co-treated with quercetin and cisplatin showed a cisplatin $\mathrm{IC}_{50}$ of $4.21 \mu \mathrm{M}$ indicating higher sensitivity compared withthe cells treated with cisplatin alone $\left(\mathrm{IC}_{50}=6.12 \mu \mathrm{M}\right)$ (Fig. 2A). In addition, 143B cells co-treated with quercetin and cisplatin exhibited significantly higher rate of

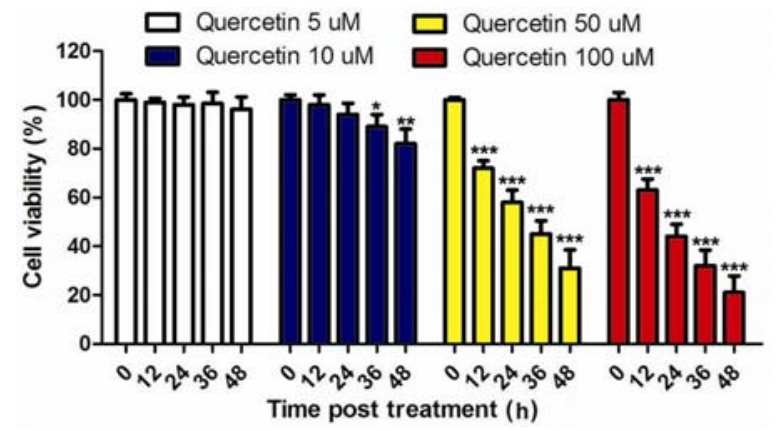

Fig. 1. Viability of $143 \mathrm{~B}$ cells in varying quercetinconcentrations.143B cells were treated in $0,5,10,50$, and $100 \mu \mathrm{M}$ quercetin concentrations. Quercetin concentration of $5 \mu \mathrm{M}$ had no significant effect on $143 \mathrm{~B}$ viability, whereas $10 \mu \mathrm{M}$ quercetin significantly reduced the cell viability at $36 \mathrm{~h}$ or $48 \mathrm{~h}$ post-treatment. Concentration of 50 or $100 \mu \mathrm{M}$ quercetin inhibited cell viability after $12 \mathrm{~h}$ of treatment.

early apoptosis $(55.2 \pm 5.61)$ compared with the cells treated with cisplatin alone $(37.3 \pm 4.67)$. Cells treated with quercetin alone showed no significant change in apoptosis rate (Figs. 2B and $2 \mathrm{C}$ ). These results demonstrated that the $5 \mu \mathrm{M}$ quercetin enhanced the cisplatin sensitivity of $143 B$ cells.

\section{Quercetin enhanced cisplatin sensitivity of 143B} osteosarcoma cells by modulating miR-217-KRAS axis Research demonstrated that miR-217 reduces cisplatin resistance by directly targeting KRAS in lung cancer cells (Guo et al., 2014). Herein, we first investigated whether a link between quercetin and miR-217-KRAS axis exists. Figure $3 \mathrm{~A}$ shows that miR-217 in 143B cells was significantly upregulated compared with that of the control cells after quercetin or cisplatin treatment; miR-217expression was much higher when co-treated with quercetin and cisplatin. In contrast with the miR-217 expression, downregulated KRAS expression on both mRNA and protein levels was observed (Fig. 3B). These results indicated that the miR-217-KRAS axis was involved in the response of osteosarcoma cells to quercetin.

To further confirm our hypothesis that quercetin enhances the cisplatin sensitivity of osteosarcoma cells by modulating the miR-217-KRAS axis, we determined the viability of cells subjected under different treatmentsaftermiR-217 knockdown and overexpression (Figs. 3C and 3D). Cells both transfected with miR-217 mimic and treated with quercetin elicited the sensitivity of osteosarcoma cells. Viability of miR-217 mimic-transfected cells treated with quercetin was significantly lower than that of the cells transfected with miR-217 mimic only or treated with quercetin alone. By contrast, osteosarcoma cells transfected with miR-217 antagomir showed resistance to cisplatin, which could be partly abated by quercetin treatment. These results demonstrated that quercetin enhanced cisplatin sensitivity of osteosarcoma cells by regulating miR-217 expression. In addition, result of Western blot analysis confirmed that miR-217 targeted KRAS of143B cells (Fig. 4A). The proliferation, migration, and invasion of $143 \mathrm{~B}$ cells were suppressed resulting from overexpression of miR-217; the opposite effects were observed when miR-217 was knocked down by antagomir (Figs. 4B-4D). These results further demonstrated that quercetin regulated the cisplatin sensitivity osteosarcoma cells by modulating the miR217-KRAS axis. 
A
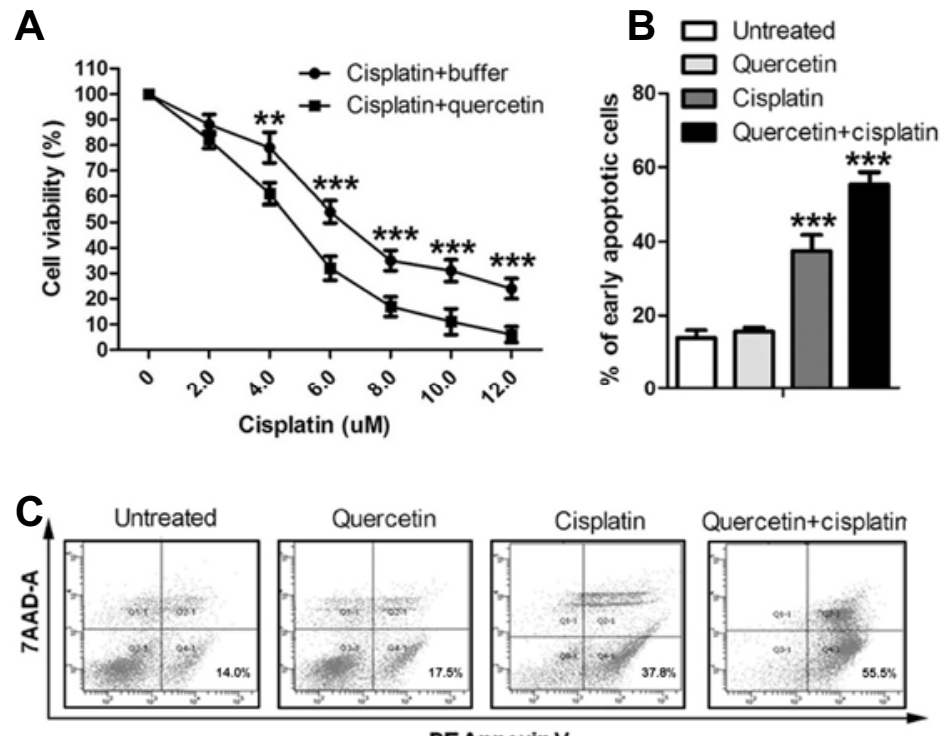

PEAnnexin V
A

C

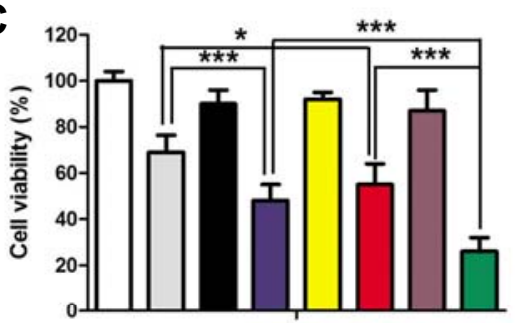

D

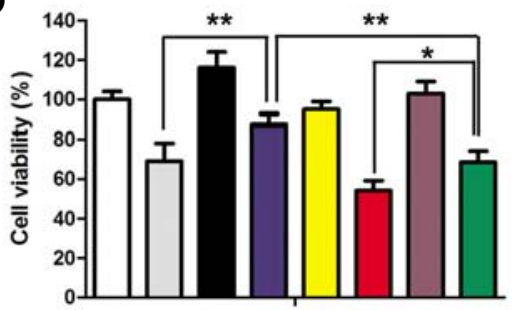

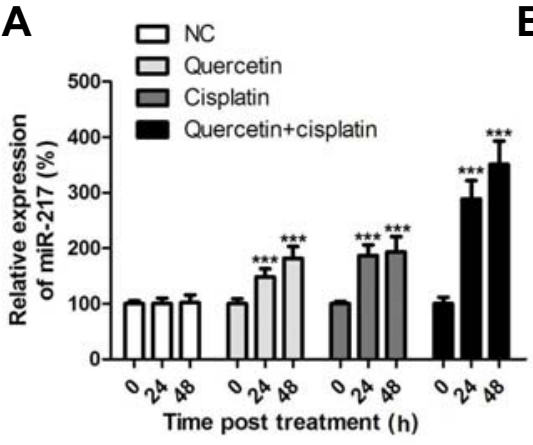

B

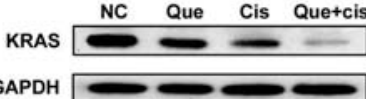

GAPDH

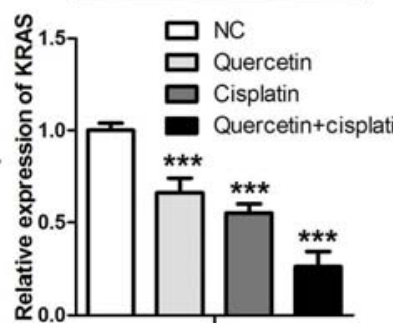

Fig. 2. Quercetin enhanced cisplatin sensitivity of $143 \mathrm{~B}$. 143B cells were treated with cisplatin at $0,2,4,6,8,10$, and $12 \mu \mathrm{M}$ for $24 \mathrm{~h}$. Quercetin was dissolved in water with $0.5 \%(\mathrm{v} / \mathrm{v})$ ethanol. Water with $0.5 \%$ ethanol was used for the control. In apoptosis assay, cells were treated with $5 \mu \mathrm{M}$ cisplatin and $5 \mu \mathrm{M}$ quercetin. (A) $143 \mathrm{~B}$ cells co-treated with $5 \mu \mathrm{M}$ quercetin and $5 \mu \mathrm{M}$ cisplatin showed a cisplatin $\mathrm{IC}_{50}$ of $4.21 \mu \mathrm{M}$, while an $\mathrm{IC}_{50}$ of $6.12 \mu \mathrm{M}$ was observed in cisplatin treatment. In "Cisplatin + quercetin" group, cells were treated with $5 \mu \mathrm{M}$ quercetin for $12 \mathrm{~h}$ before cisplatin treatment. (B, C) 143B cells co-treated with quercetin and cisplatin exhibited significant higher rate of early apoptosis $(55.2 \pm 5.61)$ compared with the cells treated with cisplatin alone $(37.3 \pm 4.67)$. No significant change in apoptosis rate was observed in cells treated with quercetin alone.
Fig. 3. Quercetin enhanced cisplatin sensitivity of 143B osteosarcoma cells by raising miR-217 level. (A) MiR217 expression upon quercetin and/or cisplatin treatment. (B) KRAS protein and mRNA expression upon quercetin and/or cisplatin treatment. (C, D) The effects of miR-217 overexpression or knockdown on cell viability under varying treatments. Cells were treated with $5 \mu \mathrm{M}$ cisplatin and $5 \mu \mathrm{M}$ quercetin for $24 \mathrm{~h}$. Quercetin was dissolved in water with $0.5 \%(\mathrm{v} / \mathrm{v})$ ethanol. Water with $0.5 \%$ ethanol was used for the control. In cell transfection, $100 \mathrm{nM}$ mimic or antagomir was used for $24 \mathrm{~h}$. Afterwards, cisplatin and/or quercetin treatments were performed.

\section{DISCUSSION}

Cisplatin is widely used as a chemotherapeutic agent, andthe effects of which on osteosarcoma have been proven (Chou et al., 2013; Martinez-Velez et al., 2014). After cisplatin treatment, many patients unfortunately experience recurrence of cancer, which become refractory to subsequent chemotherapy. This is a big challenge in chemotherapy treatment of osteosarcoma. However, cisplatin resistance in every cancer type including osteosarcoma appears to be the result of multiple and complex 



C



D
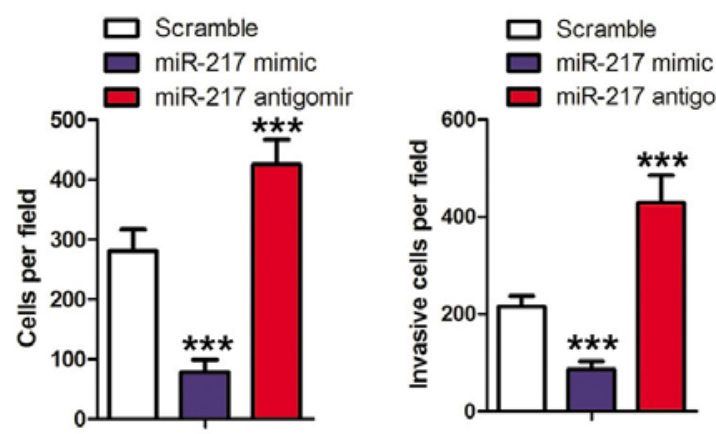

Fig. 4. miR-217 suppressed 143B cell proliferation, migration, and invasion by targeting KRAS. $100 \mathrm{nM}$ mimic or antagomir was used for cell transfection. Proliferation and migration/invasion assays were performed $24 \mathrm{~h}$ posttransfection. Approximately 5000 cells and $5 \times 10^{4}$ cells were used in the cell proliferation assay and migration/invasion assay, respectively. For Western blotting, the total proteins were extracted $48 \mathrm{~h}$ after transfection. (A) MiR-217 targeted KRAS in 143B cells. KRAS protein level was downregulated post miR-217 overexpression by mimic, while upregulated post miR-217 knocking down by antagomir. (B) MiR-217 significantly inhibited 143B proliferation. $(C, D)$ MiR-217 significantly suppressed the migration and invasion of $143 \mathrm{~B}$ cells. genetic and epigenetic factors (Galluzzi et al., 2012; Shen et al., 2012). In addition, much research focused on the modulation of cisplatin sensitivity in osteosarcoma (Wu et al., 2014). Nevertheless, there as yet exists a dearth of information on cisplatin resistance in osteosarcoma. Thus, we aimed to elucidate the mechanism of cisplatin resistance, as well as to discover the realistic method to enhance cisplatin sensitivity to improve osteosarcoma treatment.

Quercetin is a potential anticancer agent which enhances cancer chemoprevention. Synergistic anti-tumor activities of cisplatin and quercetin have long been reported in cancer treatment (Scambia et al., 1990). In vivo studies showed that quercetin modulates DNA damage induced by cisplatin in neoplastic tissue and normal tissue (Orsolic and Car, 2014). $\mathrm{PI} 3 \mathrm{~K} / \mathrm{Akt}$ pathway is a classical pathway modulating cisplatin resistance, thus influencing cisplatin resistance in osteosarcoma (Zhao et al., 2013). Through mitochondrial dysfunction and dephosphorylation of Akt, quercetin induces apoptosis in methotrexate-resistant osteosarcoma cancer cells (Xie et al., 2011).

In this study, we initially verified that the effects of quercetin on osteosarcoma depend on its working concentration. $5 \mu \mathrm{M}$ quercetin showed no inhibiting effect on osteosarcoma, but it significantly increased the cytotoxic effect of cisplatin. However, high quercetin dose $(\geq 10 \mu \mathrm{M})$ directly suppressed143B osteosarcoma cell growth, a result which is parallel with that of previous research (Berndt et al., 2013).

We previously demonstrated in our laboratory that tumor suppressor miR-217 reduces cisplatin resistance in lung cancer by negatively regulating the KRAS-Akt axis. Results in this study support the conclusion that miR-217 targets the KRAS in osteosarcoma. Interestingly, miR-217 plays contrasting roles in cancer depending on cancer type (Kato et al., 2009; Zhao et al., 2010). Herein, miR-217 is a tumor suppressor which inhibits cell proliferation and metastasis in osteosarcoma. In addition, it was demonstrated herein that miR-217 was upregulated by quercetin treatment. Meanwhile, to verify the correlation among quercetin treatment, miR-217 expression, and 143B cisplatin sensitivity, we performed rescue experiments by overexpressing or knocking down miR-217expression. Result showed that quercetin caused cisplatin sensitivity was offset by miR-217 knowndown. Overexpression of miR-217 showed similar effect as that of quercetin treatment, although higher cisplatin sensitivity was observed in 143B cells. Thus, these results confirmed that low quercetin dose $(5 \mu \mathrm{M})$ upregulated miR-217, which directly targeted KRAS that, in turn, negatively regulated the PI3K/AKT pathway, thereby suppressing cisplatin resistance in osteosarcoma. Quercetin enhances the cisplatin sensitivity of 
hepatoma cells (Kim et al., 2008). However, $5 \mu \mathrm{M}$ quercetin concentrations reduce the production of intracellular reactive oxygen species, thereby weakening the therapeutic effects of cisplatin in ovarian cancer cells (Li et al., 2014). These findings suggest that quercetin plays multiple roles depending on the type of cancer.

This study mainly demonstrated three points. First, enhanced cisplatin sensitivity in osteosarcoma cell was mediated by $5 \mu \mathrm{M}$ quercetin, while equal to or greater than $10 \mu \mathrm{M}$ quercetin directly inhibited 143B cells. Second, we proved that miR-217 is a tumor suppressor which promoted apoptosis and suppressed proliferation and metastasis of osteosarcoma cells. Third, quercetin enhanced the cisplatin sensitivity of osteosarcoma cell by modulating the miR-217-KRAS axis. These results not only elucidated the functions and mechanism of quercetin in regulating the resistance of cancer to chemotherapeutic, but also provided valuable information on a potential novel targeted drug combinations against osteosarcoma.

\section{ACKNOWLEDGMENTS}

The authors would like to thank Dr. Junhua Guo for extending excellent technical assistance and for his valuable suggestions in the design of the experiment. This study was supported by the National Natural Science Foundation of China (81371981). We declare no conflict of interest.

\section{REFERENCES}

Berndt, K., Campanile, C., Muff, R., Strehler, E., Born, W., and Fuchs, B. (2013). Evaluation of quercetin as a potential drug in osteosarcoma treatment. Anticancer Res. 33, 1297-1306.

Bielack, S.S., Carrle, D., Hardes, J., Schuck, A., and Paulussen, M. (2008). Bone tumors in adolescents and young adults. Curr. Treat Options Oncol. 9, 67-80.

Chou, A.J., Gupta, R., Bell, M.D., Riewe, K.O., Meyers, P.A., and Gorlick, R. (2013). Inhaled lipid cisplatin (ILC) in the treatment of patients with relapsed/progressive osteosarcoma metastatic to the lung. Pediatr. Blood Cancer 60, 580-586.

Galluzzi, L., Senovilla, L., Vitale, I., Michels, J., Martins, I., Kepp, O., Castedo, M., and Kroemer, G. (2012). Molecular mechanisms of cisplatin resistance. Oncogene 31, 1869-1883.

Guo, J.H., Feng, Z.J., Huang, Z., Wang, H.Y., and Lu, W.J. (2014). MicroRNA-217 functions as a tumour suppressor gene and correlates with cell resistance to cisplatin in lung cancer. Mol. Cells 37, 664-671.

Kato, M., Putta, S., Wang, M., Yuan, H., Lanting, L., Nair, I., Gunn, A., Nakagawa, Y., Shimano, H., Todorov, I., et al. (2009). TGFbeta activates Akt kinase through a microRNA-dependent amplifying circuit targeting PTEN. Nat. Cell Biol. 11, 881-U263.

Kim, J.Y., Kim, E.H., Park, S.S., Lim, J.H., Kwon, T.K., and Choi, K.S. (2008). Quercetin sensitizes human hepatoma cells to TRAIL-induced apoptosis via Sp1-mediated DR5 upregulation and proteasome-mediated c-FLIPS down-regulation. J. Cell
Biochem. 105, 1386-1398.

Li, N., Sun, C., Zhou, B., Xing, H., Ma, D., Chen, G., and Weng, D. (2014). Low concentration of quercetin antagonizes the cytotoxic effects of anti-neoplastic drugs in ovarian cancer. PLoS One 9, e100314.

Liang, W., Li, X., Li, C., Liao, L., Gao, B., Gan, H., Yang, Z., and Chen, X. (2011). Quercetin-mediated apoptosis via activation of the mitochondrial-dependent pathway in MG-63 osteosarcoma cells. Mol. Med. Rep. 4, 1017-1023.

Martinez-Velez, N., Xipell, E., Jauregui, P., Zalacain, M., Marrodan, L., Zandueta, C., Vera, B., Urquiza, L., Sierrasesumaga, L., Julian, M.S., et al. (2014). The oncolytic adenovirus Delta24RGD in combination with cisplatin exerts a potent antiosteosarcoma activity. J. Bone Miner Res. 29, 2287-2296.

Orsolic, N., and Car, N. (2014). Quercetin and hyperthermia modulate cisplatin-induced DNA damage in tumor and norma tissues in vivo. Tumour. Biol. 35, 6445-6454.

Scambia, G., Ranelletti, F.O., Benedetti Panici, P., Bonanno, G., De Vincenzo, R., Piantelli, M., and Mancuso, S. (1990). Synergistic antiproliferative activity of quercetin and cisplatin on ovarian cancer cell growth. Anticancer Drugs 1, 45-48.

Shen, D.W., Pouliot, L.M., Hall, M.D., and Gottesman, M.M. (2012) Cisplatin resistance: a cellular self-defense mechanism resulting from multiple epigenetic and genetic changes. Pharmacol. Rev. $64,706-721$.

SS, B. (2012). A review of quercetin: Antioxidant and anticancer properties. World J. Pharm. Pharm. Sci. 1, 146-160.

Suh, D.K., Lee, E.J., Kim, H.C., and Kim, J.H. (2010). Induction of $\mathrm{G}(1) / \mathrm{S}$ phase arrest and apoptosis by quercetin in human osteosarcoma cells. Arch. Pharm. Res. 33, 781-785.

Wang, P., Vadgama, J.V., Said, J.W., Magyar, C.E., Doan, N., Heber, D., and Henning, S.M. (2014). Enhanced inhibition of prostate cancer xenograft tumor growth by combining quercetin and green tea. J. Nutr. Biochem. 25, 73-80.

Wu, J., Liao, Q., He, H., Zhong, D., and Yin, K. (2014). TWIST interacts with beta-catenin signaling on osteosarcoma cell survival against cisplatin. Mol. Carcinog. 53, 440-446.

Xie, X.B., Yin, J.Q., Jia, Q., Wang, J., Zou, C.Y., Brewer, K.J., Colombo, C., Wang, Y.F., Huang, G., and Shen, J.N. (2011). Quercetin induces apoptosis in the methotrexate-resistant osteosarcoma cell line U2-OS/MTX300 via mitochondria dysfunction and dephosphorylation of Akt. Oncol. Rep. 26, 687693.

Yi, L., Zongyuan, Y., Cheng, G., Lingyun, Z., Guilian, Y., and Wei, G. (2014). Quercetin enhances apoptotic effect of tumor necrosis factor-related apoptosis-inducing ligand (TRAIL) in ovarian cancer cells through reactive oxygen species (ROS) mediated CCAAT enhancer-binding protein homologous protein (CHOP)-death receptor 5 pathway. Cancer Sci. 105, 520-527.

Zhao, W.G., Yu, S.N., Lu, Z.H., Ma, Y.H., Gu, Y.M., and Chen, J. (2010). The miR-217 microRNA functions as a potential tumor suppressor in pancreatic ductal adenocarcinoma by targeting KRAS. Carcinogenesis 31, 1726-1733.

Zhao, G.Y., Cai, C.K., Yang, T.T., Qiu, X.C., Liao, B., Li, W., Ji, Z.W., Zhao, J., Zhao, H.E., Guo, M.J., et al. (2013). MicroRNA221 induces cell survival and cisplatin resistance through PI3K/Akt pathway in human osteosarcoma. PLoS One 8, e53906. 